\title{
Parallax measurement of the Galactic Mira variables with VERA
}

\author{
A. Nakagawa ${ }^{1}$, T. Omodaka ${ }^{1}$, K. M. Shibata ${ }^{2}$, T. Kurayama ${ }^{2}$, \\ H. Imai ${ }^{1}$, S. Kameno ${ }^{1}$, M. Tsushima ${ }^{1}$, N. Matsumoto ${ }^{1}$, M. Matsui ${ }^{1}$, \\ and VERA project team ${ }^{1,2}$ \\ ${ }^{1}$ Faculty of Science, Kagoshima University, 1-21-35, Korimoto, Kagoshima, JAPAN \\ email:nakagawa@astro.sci.kagoshima-u.ac.jp \\ ${ }^{2}$ National Astronomical Observatory of Japan, 2-21-1 Osawa, Mitaka, Tokyo, JAPAN
}

\begin{abstract}
Parallax measurements of the Galactic Mira variables with VERA have started since 2004 to establish their Period-Luminosity (PL) relationship in the Galaxy. Multi-epoch VLBI observations of a semiregular variable S Crt yielded an accurate parallax of $2.27 \pm 0.14$ mas corresponding to the distance of $441_{-24}^{+29} \mathrm{pc}$. In addition to the distance, we obtained physical properties of S Crt. Temperature of the photosphere was found to be $\sim 3000 \mathrm{~K}$ by fitting the infrared spectrum with a blackbody radiation. The stellar radius was obtained based on the distance, apparent magnitude, and the temperature. Internal proper motions of circularly-arranged maser spots in S Crt were detected for the first time. Observations of the other Mira variables, such as R UMa, SY Scl, AP Lyn, and WX Psc are in progress.
\end{abstract}

Keywords. astrometry, masers, techniques: high angular resolution, techniques: interferometric, stars: AGB and post-AGB

\section{Introduction}

Mira variables are pulsating stars with periods of 100 to 1000 days. Although a narrow PL relation for Miras in the Large Magellanic Cloud was confirmed (Feast et al. 1989), the same relation for the Galactic Miras (van Leeuwen et al. 1997) has not been precisely obtained because of the ambiguity in absolute magnitudes suffering directly from inaccurate distances to each object. Using absolute magnitudes derived from accurate distances measured with VERA, we can derive a much improved PL relation for the Galactic Mira variables.

\section{Observations and data reduction}

VERA is a Japanese VLBI array dedicated to phase referencing VLBI. Synthesized beam size is typically 1.3 mas at $22 \mathrm{GHz}$. The dual beam system enables us to observe target and reference sources simultaneously, and make the data acquisition time much longer than that of conventional fast-switching VLBI. From October 2005 through May 2007, each month we observed S Crt and J1147-0724 at 22 GHz. S Crt is a semiregular variable with the pulsation periods of 155 days and have spectral class of M6E-M7E. An extragalactic source J1147-0724 is classified as a candidate source in ICRF catalog, and we used it as a positional and phase reference source. The separation between the target and a reference source is $1.23^{\circ}$ at a positional angle of $89^{\circ}$. Most likely the excess of wet atmospheric path residual at zenith causes incoherence to the reduced phase of S Crt. In order to compensate for these residuals and improve the quality of phase-referenced images, we applied trial offsets to the zenith path, ranging from -15 to $15 \mathrm{~cm}$ with an interval of $0.5 \mathrm{~cm}$ at each station (Fig. 1(b)). 

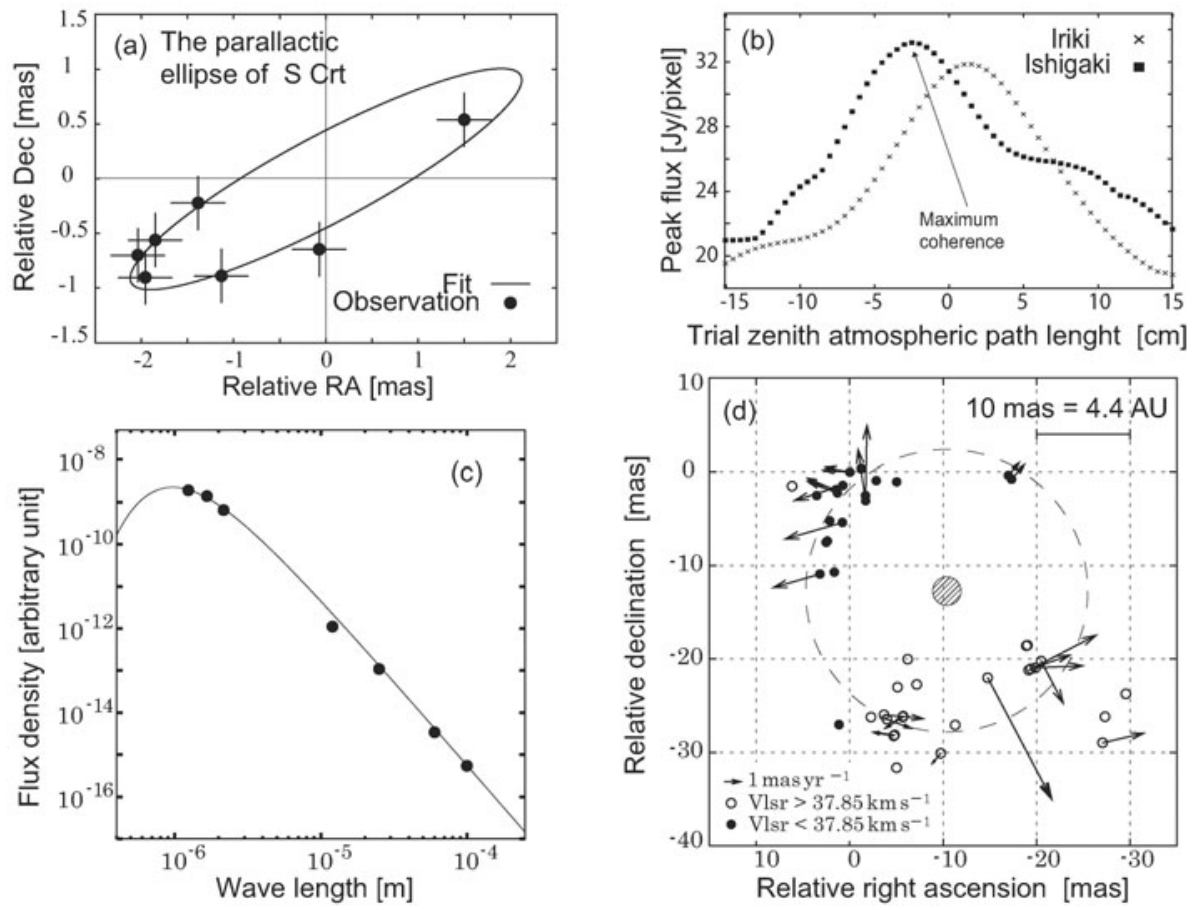

Figure 1. (a)The parallactic ellipse of S Crt. (b)We find the maximum coherence by applying trial offsets. Examples for Iriki and Ishigaki stations are presented. (c)Infrared spectrum of S Crt. The solid line is a best fit blackbody radiation obtained with the unweighted least square fitting. (d)Angular distribution and internal motions of water masers. Open and filled symbols indicate the spots showing red- and blue-shifted components relative to the stellar velocity.

\section{Result and discussion}

The annual parallax of S Crt, successfully extracted from our observations by tracing the maser spot with the LSR velocity of $34 \mathrm{mks}^{-1}$, was found to be $2.27 \pm 0.14$ mas corresponding to the distance of $441_{-24}^{+29}$ pc. Figure 1 (a) represents the parallactic ellipse. The position error of $213 \mu$ as was adopted for all observations. We estimated physical properties of S Crt based on the absolute distance measured with VERA. Infrared spectrum was well fitted with single blackbody radiation providing the temperature $\left(T_{\mathrm{bb}}\right)$ of $3000 \pm 163 \mathrm{~K}$ (Fig 1(c)). Using the K-band magnitude $\left(m_{\mathrm{K}}=0.79\right)$ and bolometric $T_{\mathrm{bb}}$, we estimated the radius of the photosphere to be $0.67 \mathrm{AU}$ shown by a shaded-circle in Fig. 1(d), along with the circularly-arranged maser spots. The radius of the maser distribution was found to be $6.6 \mathrm{AU}$ as indicated by a dashed-circle in the same figure. Arrows indicate proper motions of each spot, corresponding to the outward velocities at $6-8 \mathrm{~km} \mathrm{~s}^{-1}$. We found that $\mathrm{S}$ Crt differs from typical Mira variables. The maser distribution is more compact, the star's diameter is smaller, and the temperature is higher than those expected for typical Mira variables. Determination of the distances for larger number of samples will help to refine the Galactic PL-relation and study the difference between Miras and semiregular variables.

\section{References}

Feast, M. W., Glass, I. S., Whitelock, P. A., \& Catchpole, R. M. 1989, MNRAS 241, 375

van Leeuwen, F., Feast, M. W., Whitelock, P. A., \& Yudin, B. 1997, MNRAS 287, 955 